\title{
Rational terms in two-loop calculations
}

\author{
Stefano Pozzorini \\ Physik-Institut, Universität Zürich, CH-8057 Zürich, Switzerland \\ E-mail: pozzorinephysik.uzh.ch
}

\section{Hantian Zhang}

Physik-Institut, Universität Zürich, CH-8057 Zürich, Switzerland

E-mail: hantian.zhangephysik.uzh.ch

\section{Max F. Zoller*}

Paul Scherrer Institut, Forschungsstrasse 111, CH-5232 Villigen PSI, Switzerland

E-mail: max.zollerepsi.ch

\begin{abstract}
We present an extension of the renormalisation procedure based on the R-operation in $D$ dimensions at two-loop level, in which the numerators of all Feynman diagrams can be constructed in four dimensions, and the rational terms stemming from the interplay of $(D-4)$-dimensional numerator parts and UV poles are fully reconstructed from a finite set of universal local counterterms [1]. This represents an extension of the concept of rational terms of type $R_{2}$ to two loops. We provide a general method to compute one and two-loop rational counterterms from massive one-scale tadpole integrals. Finally, we present the full set of rational counterterms of UV origin for QED up to two-loop order.
\end{abstract}

14th International Symposium on Radiative Corrections (RADCOR2019)

9-13 September 2019

Palais des Papes, Avignon, France

${ }^{*}$ Speaker. 


\section{Introduction}

Higher-order calculations of scattering amplitudes in perturbation theory are usually performed in $D=4-2 \varepsilon$ dimensions in order to regularise divergences in Feynman integrals. In this approach, loop momenta, $\gamma$-matrices, the metric tensor and the integration measure are defined as $D$-dimensional quantities, and divergences manifest themselves as poles in $\varepsilon$. This allows for a simple Renormalisation procedure, known as the R-operation [2], which consists of the recursive subtraction of divergences stemming from all possible sub-diagrams as well as a remaining local divergence of the full diagram. The R-operation can be implemented as the insertion of counterterms into diagrams of lower loop-order.

In numerical calculations, however, vectors have to be implemented in integer dimensions. Within automated one-loop tools such as OpenLoops [3], ReCOLA [4], HelaC-1LoOp [5] or MADLOOP [6], the numerator of a Feynman integral is constructed in four dimensions, while the denominator is kept in $D$ dimensions. The difference between one-loop numerators in $D$ and four dimensions leads to a finite contribution, which is polynomial in all external momenta and internal masses, called a rational term of type $R_{2}$ [7-10].

The fully renormalised $D$-dimensional amplitude is given by

$$
\mathbf{R} \overline{\mathscr{A}}_{1, \gamma}=\overline{\mathscr{A}}_{1, \gamma}+\delta Z_{1, \gamma}
$$

where $\delta Z_{1, \gamma}$ is the UV counterterm in the $\overline{\mathrm{MS}}$-scheme. Here and in the following, an $l$-loop amplitude of a Feynman diagram $\gamma$ constructed in $D$ dimensions is denoted $\overline{\mathscr{A}}_{l, \gamma}$. An amplitude with a four-dimensional numerator, but $D$-dimensional denominator, which is the object computed in automated tools, is denoted as $\mathscr{A}_{l, \gamma}$. The renormalised $D$-dimensional amplitude can be computed from the latter, if we restore the rational contribution $\delta \mathscr{R}_{1, \gamma}$, stemming from the $(D-4)$ dimensional part of the numerator, which can be reconstructed through a counterterm insertion into a tree-level diagram, similar to the UV counterterm,

$$
\mathbf{R} \overline{\mathscr{A}}_{1, \gamma}=\mathscr{A}_{1, \gamma}+\delta Z_{1, \gamma}+\boldsymbol{\delta} \mathscr{R}_{1, \gamma} .
$$

A two-loop amplitude $\overline{\mathscr{A}}_{2, \Gamma}$ corresponding to the diagram $\Gamma$ is renormalised by first subtracting all sub-divergences and then the remaining local divergence. This is achieved by means of oneloop counterterms $\delta Z_{1, \gamma}$, associated with the divergent sub-diagrams $\gamma$, into one-loop amplitudes $\overline{\mathscr{A}}_{1, \Gamma / \gamma}$, where $\gamma$ is contracted to a point in $\Gamma$, and a local two-loop counterterm $\delta Z_{2, \Gamma}$,

$$
\mathbf{R} \overline{\mathscr{A}}_{2, \Gamma}=\overline{\mathscr{A}}_{2, \Gamma}+\sum_{\gamma} \delta Z_{1, \gamma} \cdot \overline{\mathscr{A}}_{1, \Gamma / \gamma}+\delta Z_{2, \Gamma}
$$

We generalise this procedure to

$$
\mathbf{R} \overline{\mathscr{A}}_{2, \Gamma}=\mathscr{A}_{2, \Gamma}+\sum_{\gamma}\left(\delta Z_{1, \gamma}+\delta \tilde{Z}_{1, \gamma}+\delta \mathscr{R}_{1, \gamma}\right) \cdot \mathscr{A}_{1, \Gamma / \gamma}+\left(\delta Z_{2, \Gamma}+\delta \mathscr{R}_{2, \Gamma}\right) .
$$

where all Feynman integrands on the rhs are constructed with four-dimensional numerators, and universal UV and rational counterterms. Here $\delta \mathscr{R}_{1, \gamma}$ are the well-known one-loop rational terms ${ }^{1}$

\footnotetext{
${ }^{1}$ In the literature they are usually denoted as $R_{2}$ rational terms, while we will use the symbols $\delta \mathscr{R}_{l}$, where the subscript $l$ refers to the loop order.
} 
and $\delta \tilde{Z}_{1, \gamma}$ an additional renormalisation constants proportional to $\tilde{q}^{2} / \varepsilon$, where $\tilde{q}=\bar{q}-q$ is the $(D-4)$-dimensional part of the loop momentum. We will show that $\delta \mathscr{R}_{2, \Gamma}$, which is implicitly defined in (1.4), can be computed from tadpole integrals with one auxiliary mass scale and that it is indeed a rational term. Formula (1.4) can directly be implemented in numerical algorithms and hence serves as an important step towards two-loop automation. In this discussion we restrict ourselves to rational terms of UV origin. A detailed description of our method and results can be found in [1].

\section{Rational terms at one loop}

\subsection{One loop rational terms from massive tadpoles with one scale}

Let us consider the amplitude of a one-particle irreducible one-loop diagram $\gamma$,

$$
\overline{\mathscr{A}}_{1, \gamma}=\overline{\bar{D}}_{0}(\underbrace{\bar{D}_{N-1}}_{\bar{D}_{1}}=\int \mathrm{d} \bar{q}_{1} \frac{\overline{\mathscr{N}}\left(\bar{q}_{1}\right)}{\bar{D}_{0}\left(\bar{q}_{1}\right) \cdots D_{N-1}\left(\bar{q}_{1}\right)},
$$

with $\mathrm{d} \bar{q}_{1}=\left(\mu_{\overline{\mathrm{MS}}}\right)^{2 \varepsilon} \mathrm{d}^{d} \bar{q}_{1} /(2 \pi)^{d}$, where the scalar denominators are defined as

$$
D_{k}\left(\bar{q}_{1}\right)=\left(\bar{q}_{1}+p_{k}\right)^{2}-m_{k}^{2}
$$

with external momenta $p_{k}$ and masses $m_{k}$. We mark $D$-dimensional objects, such as loop momenta, $\gamma$-matrices and the metric, as well as their $D$-dimensional Lorentz indices with a bar, i.e. $\bar{q}, \bar{\gamma}^{\bar{\mu}}, \bar{g}^{\bar{\mu}} \bar{v}$, while their projections to four dimensions are are denoted $q, \gamma^{\mu}$ and $g^{\mu \nu}$. The same applies to the numerator $\bar{N}(\bar{q})$ of a Feynman integrand constructed from $D$-dimensional quantities or $\mathscr{N}(q)$ in four dimensions. ${ }^{2}$ The dimension of the numerator is denoted $D_{\mathrm{n}} \in\{D, 4\}$.

In $D_{\mathrm{n}}=D$ dimensions, the numerator $\mathscr{N}\left(\bar{q}_{1}\right)$ can be split into a four-dimensional part

$$
\mathscr{N}\left(q_{1}\right)=\left.\overline{\mathscr{N}}\left(\bar{q}_{1}\right)\right|_{\bar{g} \rightarrow g, \bar{\gamma} \rightarrow \gamma, \bar{q}_{1} \rightarrow q_{1}}
$$

and a remnant

$$
\tilde{N}\left(\bar{q}_{1}\right)=\overline{\mathscr{N}}\left(\bar{q}_{1}\right)-\mathscr{N}\left(q_{1}\right)=\mathscr{O}\left(\varepsilon, \tilde{q}_{1}\right) .
$$

The rational term in (1.2) corresponds to

$$
\delta \mathscr{R}_{1, \gamma}=\overline{\mathscr{A}}_{1, \gamma}-\mathscr{A}_{1, \gamma}=\int \mathrm{d} \bar{q}_{1} \frac{\tilde{\mathscr{N}}\left(\bar{q}_{1}\right)}{D_{0}\left(\bar{q}_{1}\right) \cdots D_{N-1}\left(\bar{q}_{1}\right)},
$$

which generates finite terms through the interplay of the $(D-4)$-dimensional numerator with the $1 / \varepsilon$ UV poles. ${ }^{3}$ Hence, such $\tilde{\mathscr{N}}$-contributions arise only in UV divergent 1PI diagrams.

\footnotetext{
${ }^{2}$ In the 't Hooft-Veltman scheme, external momenta $p_{i}$ are always defined in four dimensions.

${ }^{3}$ At one loop $1 / \varepsilon$ poles of IR origin do not generate rational terms. See App. A of [17].
} 
We now discuss a well-established method [11,12] for the calculation of the UV divergent part of a Feynman diagram from massive tadpoles with one scale, and adapt it to the calculation of rational terms. This method is based on the exact decomposition of all propagator denominators,

$$
\begin{aligned}
\frac{1}{D_{i}} & =\frac{1}{\bar{q}_{1}^{2}-M^{2}}+\frac{\Delta_{i}}{\bar{q}_{1}^{2}-M^{2}} \frac{1}{D_{i}} \\
\longrightarrow & =\longrightarrow
\end{aligned}
$$

with

$$
\Delta_{k}\left(\bar{q}_{1}\right)=\left(\bar{q}_{1}^{2}-M^{2}\right)-D_{k}\left(\bar{q}_{1}\right)=-2 \bar{q}_{1} \cdot p_{k}-p_{k}^{2}+m_{k}^{2}-M^{2},
$$

where $M^{2}$ is an auxiliary squared mass scale. We have introduced a graphical representation, in which thick red lines depict the pure tadpole propagators $\left(\bar{q}_{1}^{2}-M^{2}\right)^{-2}$ and black ones to the original propagators $D_{i}$. This can be used recursively up to a fixed order $X+1$ in the tadpole propagators, in order to isolate the UV divergences and rational terms in pure tadpole integrals with one auxiliary scale $M^{2}$.

For a more compact notation, we introduce two operators $\mathbf{S}_{X}^{(1)}$ and $\mathbf{F}_{X}^{(1)}$, which generate, respectively, the pure tadpole terms up to order $X$ in a naive $1 / q$ power counting of the integrand, and the higher-order remnant containing all original propagators, ${ }^{4}$

$$
\frac{1}{D_{k}\left(\bar{q}_{1}\right)}=\left(\mathbf{S}_{X}^{(1)}+\mathbf{F}_{X}^{(1)}\right) \frac{1}{D_{k}\left(\bar{q}_{1}\right)},
$$

with

$$
\mathbf{S}_{X}^{(1)} \frac{1}{D_{k}\left(\bar{q}_{1}\right)}=\sum_{\sigma=0}^{X} \frac{\left[\Delta_{k}\left(\bar{q}_{1}\right)\right]^{\sigma}}{\left(\bar{q}_{1}^{2}-M^{2}\right)^{\sigma+1}}, \quad \mathbf{F}_{X}^{(1)} \frac{1}{D_{k}\left(\bar{q}_{1}\right)}=\frac{\left[\Delta_{k}\left(\bar{q}_{1}\right)\right]^{X+1}}{\left(\bar{q}_{i}^{2}-M^{2}\right)^{X+1}} \frac{1}{D_{k}\left(\bar{q}_{1}\right)} .
$$

If $X$ is chosen as the superficial degree of divergence and (2.9) applied to every propagator denominator along the loop, all terms containing an original $D_{k}$ are free from UV divergences.

For a full chain of propagators these operators are defined to give

$$
\mathbf{S}_{X}^{(1)} \frac{1}{D_{0}\left(\bar{q}_{1}\right) \cdots D_{N-1}\left(\bar{q}_{1}\right)}=\sum_{\sigma=0}^{X} \frac{\Delta^{(\sigma)}\left(\bar{q}_{1}\right)}{\left(\bar{q}_{1}^{2}-M^{2}\right)^{N+\sigma}}, \quad \mathbf{F}_{X}^{(1)}=1-\mathbf{S}_{X}^{(1)},
$$

where

$$
\Delta^{(\sigma)}\left(\bar{q}_{1}\right)=\left.\sum_{\sigma_{0}=0}^{\sigma} \ldots \sum_{\sigma_{0}=N-1}^{\sigma} \prod_{k=0}^{N-1}\left[\Delta_{k}\left(\bar{q}_{1}\right)\right]^{\sigma_{k}}\right|_{\sigma_{0}+\cdots+\sigma_{N-1}=\sigma}
$$

is a polynomial in the external momenta, the masses and the loop momentum. Since the finite terms collected by $\mathbf{F}_{X}^{(1)}$ cancel in the difference (1.2), we can compute the rational term of type $R_{2}$ as

$$
\delta \mathscr{R}_{1, \gamma}=\mathbf{S}_{X}^{(1)}\left(\overline{\mathscr{A}}_{1, \gamma}-\mathscr{A}_{1, \gamma}\right)=\sum_{\sigma=N}^{N+X} \int \mathrm{d} \bar{q}_{1} \frac{\left(\overline{\mathscr{N}}\left(\bar{q}_{1}\right)-\mathscr{N}\left(q_{1}\right)\right) \Delta^{(\sigma)}\left(\bar{q}_{1}\right)}{\left(\bar{q}_{1}^{2}-M^{2}\right)^{\sigma}} .
$$

\footnotetext{
${ }^{4}$ The label $(i)$ in $\mathbf{S}_{X}^{(i)}$ and $\mathbf{F}_{X}^{(i)}$ refers to the chain of propagator denominators with loop momentum $\bar{q}_{i}$, on which these operators act exclusively, since in multi-loop diagrams there are several chains with different $\bar{q}_{i}$.
} 


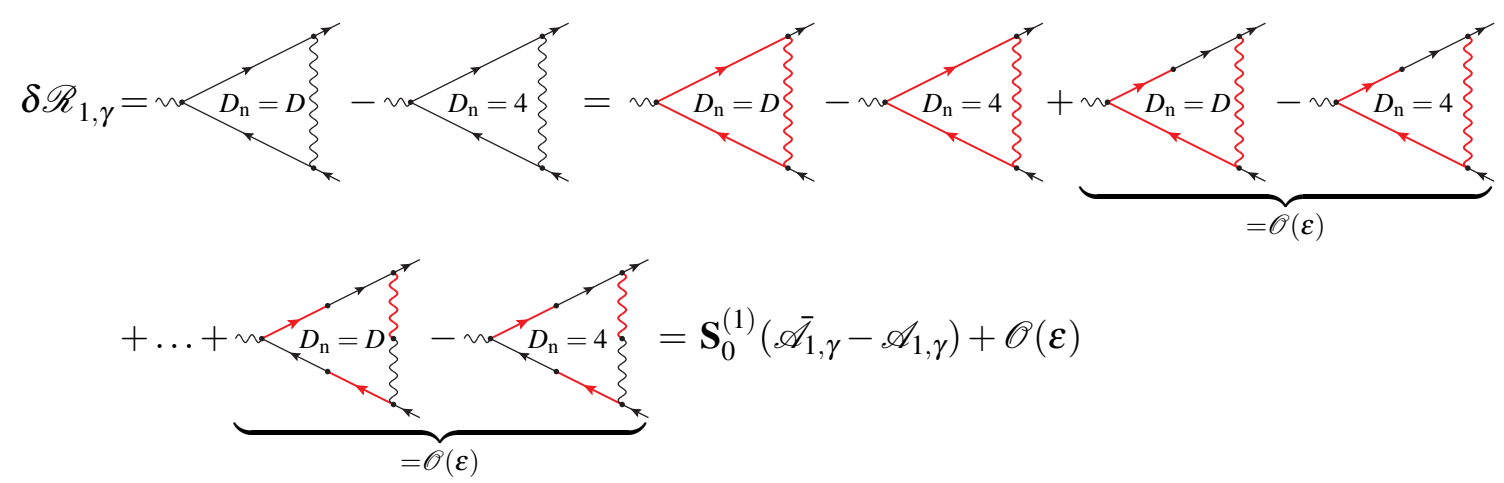

Figure 1: $\tilde{\mathscr{N}}$-contribution for the one-loop vertex correction in QED. $D_{\mathrm{n}}$ is the numerator dimension.

The numerator in (2.1) can be written in terms of loop momentum tensors up to rank $R$,

$$
\overline{\mathscr{N}}\left(\bar{q}_{1}\right)=\sum_{r=0}^{R} \overline{\mathscr{N}}_{\bar{\mu}_{1} \cdots \bar{\mu}_{r}} \bar{q}_{1}^{\bar{\mu}_{1}} \cdots \bar{q}_{1}^{\bar{\mu}_{r}}
$$

Decomposing the extended numerators and including the resulting term (2.13) into the loop numerator yields

$$
\overline{\mathscr{N}}\left(\bar{q}_{1}\right) \Delta^{(\sigma)}\left(\bar{q}_{1}\right)=\sum_{r=0}^{R+\sigma} \overline{\mathscr{N}}_{\bar{\mu}_{1} \cdots \bar{\mu}_{r}}^{(\sigma)} \bar{q}_{1}^{\bar{\mu}_{1}} \cdots \bar{q}_{1}^{\bar{\mu}_{r}}, \quad \mathscr{N}\left(q_{1}\right) \Delta^{(\sigma)}\left(\bar{q}_{1}\right)=\sum_{r=0}^{R+\sigma} \mathscr{N}_{\mu_{1} \cdots \mu_{r}}^{(\sigma)} q_{1}^{\mu_{1}} \cdots q_{1}^{\mu_{r}}
$$

Hence, we can compute the $\tilde{\mathscr{N}}$-contributions from coefficients constructed in $D$ and four dimensions and fully $D$-dimensional tensor integrals,

$$
\delta \mathscr{R}_{1, \gamma}=\sum_{\sigma=0}^{X} \sum_{r=0}^{R+\sigma}\left(\bar{N}_{\bar{\mu}_{1} \cdots \bar{\mu}_{r}}^{(\sigma)}-\mathscr{N}_{\mu_{1} \cdots \mu_{r}}^{(\sigma)}\right) \int \mathrm{d} \bar{q} \frac{\bar{q}^{\bar{\mu}_{1}} \cdots \bar{q}^{\bar{\mu}_{r}}}{\left(\bar{q}^{2}-M^{2}\right)^{n}}
$$

using $\bar{q} \cdot p_{j}=q \cdot p_{j}$ for external four-momenta $p_{j}$ and $A_{\mu_{i}} B^{\bar{\mu}_{i}}=A_{\mu_{i}} B^{\mu_{i}}$ for any vectors $A, B$. Since the denominator decomposition (2.9) is exact and $M^{2}$-independent, and $\mathbf{F}_{X}^{(1)}\left(\overline{\mathscr{A}}_{1, \gamma}-\mathscr{A}_{1, \gamma}\right)=\mathscr{O}(\varepsilon)$, the finite part of $\mathbf{S}_{X}^{(1)}\left(\overline{\mathscr{A}}_{1, \gamma}-\mathscr{A}_{1, \gamma}\right)$ and hence $\delta \mathscr{R}_{1, \gamma}$ does also not depend on $M^{2}$. The dependence on external momenta and masses resides exclusively in the $\overline{\mathscr{N}}_{\bar{\mu}_{1} \cdots \bar{\mu}_{r}}^{(\sigma)}$ and $\mathscr{N}_{\mu_{1} \cdots \mu_{r}}^{(\sigma)}$ in polynomial form, which proves that $\delta \mathscr{R}_{1, \gamma}$ is indeed a rational term. We illustrate the cancellation of terms with original propagators and the resulting pure tadpole contribution for the one-loop vertex correction in QED in Fig. 1.

\subsection{One-loop sub-diagrams with numerator dimension $D_{\mathrm{n}}=4$}

In the case of a one-loop sub-diagram with loop momentum $\bar{q}_{1}$ to a two-loop diagram, we encounter a subtlety, namely the fact that one external momentum flowing through the sub-diagram is the second loop momentum $\bar{q}_{2}$, which is $D$-dimensional in the construction of the numerator $\bar{N}\left(\bar{q}_{1}, \bar{q}_{2}\right)$ and four-dimensional in the construction of $\mathscr{N}\left(q_{1}, q_{2}\right)$, but always $D$-dimensional in the denominators. This requires an extension of (1.2) in order to subtract all divergences arising in $\mathscr{A}_{1, \gamma}\left(q_{2}\right)$. 
$\begin{aligned} \mathbf{R}\left[\sum_{\bar{\alpha}_{2}}^{\alpha_{D_{\mathrm{n}}=D}}\right]_{\bar{\alpha}_{2}}^{\bar{\alpha}_{1}} & =\left[\sum_{\bar{\alpha}_{2}}^{\bar{\alpha}_{1}} \delta Z_{1, \gamma}\left(\bar{q}_{2}\right)\right]_{D_{\mathrm{n}}=D} \\ & =\left[\sum_{\alpha_{2}}^{\bar{\alpha}_{1}}\left(\delta Z_{1, \gamma}\left(q_{2}\right)+\delta \tilde{Z}_{1, \gamma}\left(\tilde{q}_{2}\right)+\delta \mathscr{R}_{1, \gamma}\left(q_{2}\right)\right)\right]_{D_{\mathrm{n}}=4}+\mathscr{O}(\varepsilon)\end{aligned}$

Figure 2: Graphical representation for a renormalised sub-diagram in numerator dimensions $D_{\mathrm{n}}=D$ and $D_{\mathrm{n}}=4$ for the case of a QED self-energy. The $D$-dimensional external momentum $\bar{q}_{2}$ is decomposed into a four-dimensional part $q_{2}$ and a $(D-4)$-dimensional one $\tilde{q}_{2}=\bar{q}_{2}-q_{2}$.

In this section we therefore consider the renormalisation of $\overline{\mathscr{A}}_{1, \gamma}\left(\bar{q}_{2}\right)$ and its variant $\mathscr{A}_{1, \gamma}\left(q_{2}\right)$, where the argument refers to the momentum in the numerator of the integrand. The denominators are in both cases constructed with $\bar{q}_{2}$ in $D$ dimensions.

In the case of a fully $D$-dimensional one-loop amplitude the UV counterterm in the $\overline{\mathrm{MS}}$ scheme, introduced in (1.1), is derived as ${ }^{5}$

$$
\delta Z_{1, \gamma}\left(\bar{q}_{2}\right)=-\mathbf{K} \overline{\mathscr{A}}_{1, \gamma}\left(\bar{q}_{2}\right)
$$

where the operator $\mathbf{K}$ performs a Laurent expansion in $\varepsilon$ and discards all terms of order $\varepsilon^{0}$ and higher, isolating the pole part of an amplitude. We compute the UV counterterm for the same amplitude with a four-dimensional numerator as

$$
\begin{aligned}
\mathbf{K} \mathscr{A}_{1, \gamma}\left(q_{2}\right) & =\mathbf{K} \mathbf{S}_{X}^{(1)} \mathscr{A}_{1, \gamma}\left(q_{2}\right)=\mathbf{K} \sum_{r=0}^{R} \mathscr{N}_{\mu_{1} \ldots \mu_{r}}\left(q_{2}\right) \sum_{\sigma=0}^{X} \int \mathrm{d} \bar{q}_{1} \frac{\bar{q}_{1}^{\bar{\mu}_{1}} \cdots \bar{q}_{1}^{\bar{\mu}_{r}} \Delta^{(\sigma)}\left(\bar{q}_{1}, \bar{q}_{2}\right)}{\left(\bar{q}_{1}^{2}-M^{2}\right)^{N+\sigma}} \\
& =-\delta Z_{1, \gamma}\left(q_{2}\right)-\delta \tilde{Z}_{1, \gamma}\left(\tilde{q}_{2}\right)
\end{aligned}
$$

where the first term on the rhs is the usual $\overline{\mathrm{MS}}$ counterterm with $\bar{q}_{2} \rightarrow q_{2}$, and the $D$-dimensional $\bar{q}_{2}=q_{2}+\tilde{q}_{2}$ in the extended numerator leads to an additional counterterm $\delta \tilde{Z}_{1, \gamma}\left(\tilde{q}_{2}\right) \propto \tilde{q}_{2}^{2} / \varepsilon$ stemming from scalar products $\tilde{q}_{1} \tilde{q}_{2}$ and $\tilde{q}_{2}^{2}$ in $\Delta^{(\sigma)}$ for $X \geq 2$. This is a direct consequence of the fact that scalar denominators are still $D$-dimensional. Hence the renormalised amplitude, i.e. one with all poles in $\varepsilon$ subtracted, in $D_{\mathrm{n}}=4$ can be defined as

$$
\mathbf{R} \mathscr{A}_{1, \gamma}\left(q_{2}\right):=(1-\mathbf{K}) \mathscr{A}_{1, \gamma}\left(q_{2}\right)=\mathscr{A}_{1, \gamma}\left(q_{2}\right)+\delta Z_{1, \gamma}\left(q_{2}\right)+\delta \tilde{Z}_{1, \gamma}\left(\tilde{q}_{2}\right)
$$

and the renormalised $D$-dimensional amplitude can be written as

$$
\mathbf{R} \overline{\mathscr{A}}_{1, \gamma}\left(\bar{q}_{2}\right)=\mathbf{R} \mathscr{A}_{1, \gamma}\left(q_{2}\right)+\boldsymbol{\delta} \mathscr{R}_{1, \gamma}\left(q_{2}\right)+\mathscr{O}(\varepsilon, \tilde{q})
$$

with the well-known one-loop rational term of type $R_{2}$ [1].

In renormalisable theories, $X \geq 2$ is only fulfilled for self-energies, in QED only for the photon self-energy. The renormalisation procedure for this case is depicted in Fig. 2. In all other cases $\delta \tilde{Z}_{1, \gamma}\left(\tilde{q}_{2}\right)=0$.

\footnotetext{
${ }^{5}$ We suppress the indices $\alpha_{1}, \alpha_{2}$ connecting the sub-diagram to the rest of the two-loop diagram, $\overline{\mathscr{A}}_{1, \gamma}=\mathscr{\mathscr { A }}_{1, \gamma} \bar{\alpha}_{1} \bar{\alpha}_{2}$, etc.
} 


\section{Rational terms at two loops}

A generic irreducible two-loop diagram ${ }^{6} \Gamma$ can be decomposed into three chains, each consisting of a numerator $\overline{\mathscr{N}}^{(i)}\left(\bar{q}_{i}\right)$ and a denominator

$$
\mathscr{D}^{(i)}\left(\bar{q}_{i}\right)=D_{0}^{(i)}\left(\bar{q}_{i}\right) \cdots D_{N_{i}-1}^{(i)}\left(\bar{q}_{i}\right), \quad \text { with } \quad D_{a}^{(i)}\left(\bar{q}_{i}\right)=\left(\bar{q}_{i}-p_{i a}\right)^{2}-m_{i a}^{2},
$$

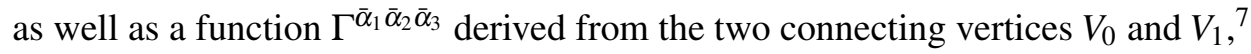

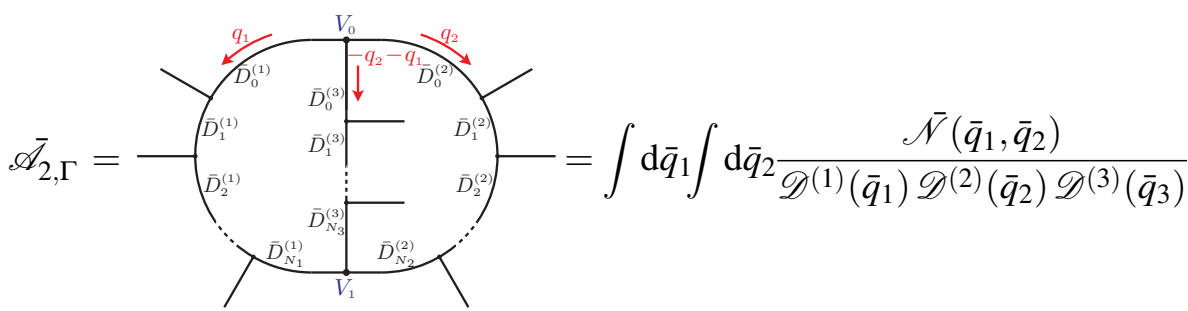

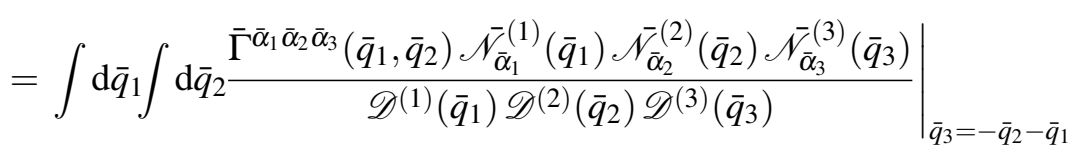

There are three sub-diagrams $\gamma_{i}$, each constructed from two chains $j, k$ and the connecting function

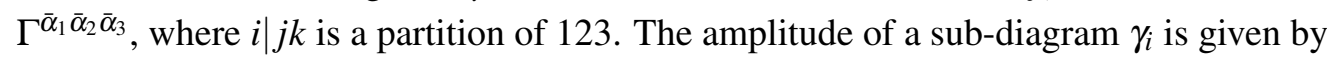

$$
\mathscr{\mathscr { A }}_{1, \gamma_{i}}^{\bar{\alpha}_{i}}\left(\bar{q}_{i}\right)=\left.\int \mathrm{d} \bar{q}_{j} \frac{\bar{\Gamma}^{\bar{\alpha}_{1} \bar{\alpha}_{2} \bar{\alpha}_{3}} \overline{\mathscr{N}}_{\bar{\alpha}_{j}}^{(j)}\left(\bar{q}_{j}\right) \overline{\mathscr{N}}_{\bar{\alpha}_{k}}^{(k)}\left(\bar{q}_{k}\right)}{\mathscr{D}^{(j)}\left(\bar{q}_{j}\right) \mathscr{D}^{(k)}\left(\bar{q}_{k}\right)}\right|_{\bar{q}_{k}=-\bar{q}_{i}-\bar{q}_{j}},
$$

and the insertion into its complement $\Gamma / \gamma_{i}$ can be written as

$$
\overline{\mathscr{A}}_{2, \Gamma}=\overline{\mathscr{A}}_{1, \gamma_{i}} \cdot \overline{\mathscr{A}}_{1, \Gamma / \gamma_{i}}=\int \mathrm{d} \bar{q}_{i} \overline{\mathscr{A}}_{1, \gamma_{i}} \bar{\alpha}_{i}\left(\bar{q}_{i}\right) \frac{\overline{\mathscr{N}}_{\bar{\alpha}}^{(i)}\left(\bar{q}_{i}\right)}{\mathscr{D}^{(i)}\left(\bar{q}_{i}\right)} .
$$

Formula (1.4), which constructs a renormalised $D$-dimensional amplitude from ingredients with four-dimensional numerators and insertions of UV counterterms and known one-loop rational terms, implicitly defines the yet unknown remnant $\delta \mathscr{R}_{2, \Gamma}$ as

$$
\delta \mathscr{R}_{2, \Gamma}=\left(\overline{\mathscr{A}}_{2, \Gamma}+\sum_{\gamma} \delta Z_{1, \gamma} \cdot \overline{\mathscr{A}}_{1, \Gamma / \gamma}\right)-\left(\mathscr{A}_{2, \Gamma}+\sum_{\gamma}\left(\delta Z_{1, \gamma}+\delta \tilde{Z}_{1, \gamma}+\delta \mathscr{R}_{1, \gamma}\right) \cdot \mathscr{A}_{1, \Gamma / \gamma}\right)
$$

We will show in the following that $\delta \mathscr{R}_{2, \Gamma}$ is polynomial in all external momenta and masses, which makes it a rational term that can be written as a local counterterm.

We distinguish two cases, diagrams with superficial degree of divergence $X(\Gamma)<0$ (no global divergence) and diagrams with $X(\Gamma)>0$ (global divergence).

\footnotetext{
${ }^{6}$ Reducible diagrams can be computed by applying the previous discussion to each one-loop sub-diagram $\gamma_{1}, \gamma_{2}$ separately, since

$$
\mathbf{R} \overline{\mathscr{A}}_{2, \Gamma}=\mathbf{R} \overline{\mathscr{A}}_{1, \gamma_{1}} \cdot \mathbf{R} \overline{\mathscr{A}}_{1, \gamma_{2}}
$$

${ }^{7}$ The multi-indices $\bar{\alpha}_{i}$ consist of two Lorentz or spinor indices.
} 


\subsection{Diagrams without global divergence}

Two-loop diagrams with $X(\Gamma)<0$ can have at most one divergent sub-diagram $\gamma_{i}$ (see e.g. [1]), i.e. a sub-diagram with $X\left(\gamma_{i}\right) \geq 0$. The renormalised amplitude in $D_{\mathrm{n}}=d$ dimensions is given as

$$
\mathbf{R} \overline{\mathscr{A}}_{2, \Gamma}=\overline{\mathscr{A}}_{2, \Gamma}-\left(\mathbf{K} \overline{\mathscr{A}}_{1, \gamma_{i}}\right) \cdot \overline{\mathscr{A}}_{1, \Gamma / \gamma_{i}}=\left(\overline{\mathscr{A}}_{1, \gamma_{i}}+\delta Z_{1, \gamma_{i}}\right) \cdot \overline{\mathscr{A}}_{1, \Gamma / \gamma_{i}}
$$

Since the sub-diagram $\gamma_{i}$ plus its UV counterterm, and also its counterpart $\Gamma / \gamma_{i}$ are free from any divergence we can discard any $\mathscr{O}(\varepsilon)$ contributions both in the renormalised sub-diagram $\gamma_{i}$ and $\Gamma / \gamma_{i}$. Hence, we construct the numerator of $\Gamma / \gamma_{i}$ in $D_{\mathrm{n}}=4$, leading to ${ }^{8}$

$$
\mathbf{R} \overline{\mathscr{A}}_{2, \Gamma}=\left(\overline{\mathscr{A}}_{1, \gamma_{i}}+\delta Z_{1, \gamma_{i}}\right) \cdot \mathscr{A}_{1, \Gamma / \gamma_{i}}+\mathscr{O}(\varepsilon) .
$$

The UV-subtracted $\gamma_{i}$ can be expressed with its amplitude constructed in $D_{\mathrm{n}}=4$ using (2.21),

$$
\mathbf{R} \overline{\mathscr{A}}_{2, \Gamma}=\left(\mathscr{A}_{1, \gamma_{i}}+\delta Z_{1, \gamma_{i}}+\delta \tilde{Z}_{1, \gamma_{i}}+\delta \mathscr{R}_{1, \gamma_{i}}\right) \cdot \mathscr{A}_{1, \Gamma / \gamma_{i}}+\mathscr{O}(\varepsilon)
$$

which means that for $X(\Gamma)<0$ we find

$$
\delta \mathscr{R}_{2, \Gamma}=0 .
$$

This shows that non-vanishing two-loop rational terms of UV origin occur only in the finite set of diagrams with a global divergence.

\subsection{Diagrams with global divergence}

In this section we show that $\delta \mathscr{R}_{2, \Gamma}$ in (1.4) is also a rational term for $X(\Gamma) \geq 0$. Our starting point is the difference (3.5), which gives the remaining $\tilde{\mathscr{N}}$-contribution after the subtraction of the sub-divergences from $\overline{\mathscr{A}}_{2, \Gamma}$ and $\mathscr{A}_{2, \Gamma}$, and the restoration of the $\tilde{\mathscr{N}}$-contributions stemming from the one-loop sub-diagrams of $\Gamma$. We show that (3.5) can be fully computed from massive tadpoles with one auxiliary scale $M^{2}$, of which the result is independent. We then conclude, similar to the one-loop case, that (3.5) is polynomial in the external momenta and masses, and hence a rational term.

Our strategy is to apply a tadpole decomposition (2.11) to the three chains in $\overline{\mathscr{A}}_{2, \Gamma}$ and $\mathscr{A}_{2, \Gamma}$ and their subtraction terms to high enough powers in $1 / q_{i}$, such that all terms captured by the operators $\mathbf{F}_{X_{i}}^{(i)}$, in particular all terms involving original propagators, are free from global divergences and hence cancel exactly in (3.5) according to (3.9).

To this end we express the rhs of (3.5) with the linear operators $\mathbf{K}$ and $\tilde{\mathbf{K}}$,

$$
\left(\overline{\mathscr{A}}_{2, \Gamma}+\sum_{i}\left(-\mathbf{K} \overline{\mathscr{A}}_{1, \gamma_{i}}\right) \cdot \overline{\mathscr{A}}_{1, \Gamma / \gamma_{i}}\right)-\left(\mathscr{A}_{2, \Gamma}+\sum_{i}\left((-\mathbf{K}+\tilde{\mathbf{K}}) \mathscr{A}_{1, \gamma_{i}}\right) \cdot \mathscr{A}_{1, \Gamma / \gamma}\right)=: \tilde{\mathbf{K}}_{\mathrm{loc}} \overline{\mathscr{A}}_{2, \Gamma}
$$

where

$$
\tilde{\mathbf{K}} \mathscr{A}_{1, \gamma_{i}}:=\mathbf{R} \overline{\mathscr{A}}_{1, \gamma_{i}}-\mathbf{R} \mathscr{A}_{1, \gamma_{i}} \equiv \boldsymbol{\delta} \mathscr{R}_{1, \gamma_{i}} .
$$

The operator $\tilde{\mathbf{K}}_{\text {loc }}$, which constructs $\delta \mathscr{R}_{2, \Gamma}$ is in turn linear. The divergent parts of a two-loop diagram and its three sub-diagrams can be isolated by applying independent tadpole decompositions

\footnotetext{
${ }^{8}$ In this step, we also project the Lorentz indices connecting $\gamma_{i}$ and $\Gamma / \gamma_{i}$ to four dimensions, $\bar{\alpha}_{i} \rightarrow \alpha_{i}$.
} 
(2.11) to the three chains. Each chain $i$ is expanded to the maximum of the global divergence of $\Gamma$ and the divergences of the sub-diagrams $\gamma_{j}, \gamma_{k}$ of which it is a part, ${ }^{9}$

$$
X_{i}(\Gamma)=\max \left\{X(\Gamma), X\left(\gamma_{j}\right), X\left(\gamma_{k}\right)\right\} .
$$

Applying this operation to (3.10) gives

$$
\begin{aligned}
\tilde{\mathbf{K}}_{\mathrm{loc}} \overline{\mathscr{A}}_{2, \Gamma} & =\tilde{\mathbf{K}}_{\mathrm{loc}}\left(\mathbf{S}_{X_{1}}^{(1)}+\mathbf{F}_{X_{1}}^{(1)}\right)\left(\mathbf{S}_{X_{2}}^{(2)}+\mathbf{F}_{X_{2}}^{(2)}\right)\left(\mathbf{S}_{X_{3}}^{(3)}+\mathbf{F}_{X_{3}}^{(3)}\right) \overline{\mathscr{A}}_{2, \Gamma} \\
& =\tilde{\mathbf{K}}_{\mathrm{loc}} \overline{\mathscr{A}}_{2, \Gamma_{\text {tad }}}+\tilde{\mathbf{K}}_{\mathrm{loc}} \overline{\mathscr{A}}_{2, \Gamma_{\mathrm{rem}}},
\end{aligned}
$$

with a pure tadpole term and remnant terms

$$
\begin{aligned}
& \overline{\mathscr{A}}_{2, \Gamma_{\text {tad }}}=\mathbf{S}_{X_{1}}^{(1)} \mathbf{S}_{X_{2}}^{(2)} \mathbf{S}_{X_{3}}^{(3)} \overline{\mathscr{A}}_{2, \Gamma}, \\
& \overline{\mathscr{A}}_{2, \Gamma_{\text {rem }}}=\mathbf{F}_{X_{1}}^{(1)} \mathbf{F}_{X_{2}}^{(2)} \mathbf{F}_{X_{3}}^{(3)} \overline{\mathscr{A}}_{2, \Gamma}+\sum_{i=1}^{3} \mathbf{S}_{X_{i}}^{(i)} \mathbf{F}_{X_{j}}^{(j)} \mathbf{F}_{X_{k}}^{(k)} \overline{\mathscr{A}}_{2, \Gamma}+\sum_{i=1}^{3} \mathbf{F}_{X_{i}}^{(i)} \mathbf{S}_{X_{j}}^{(j)} \mathbf{S}_{X_{k}}^{(k)} \overline{\mathscr{A}}_{2, \Gamma} .
\end{aligned}
$$

All terms that enter (3.16) involve at least one $\mathbf{F}$ operator, which reduces the superficial degree of divergence $X(\Gamma)$, as well as $X\left(\gamma_{j}\right)$ and $X\left(\gamma_{k}\right)$, by $X_{i}(\Gamma)+1$. Thus the remnant part (3.16) is free from global divergences, and the discussion in section 3.1 implies $^{10}$

$$
\delta \mathscr{R}_{2, \Gamma_{\text {rem }}}=\tilde{\mathbf{K}}_{\text {loc }} \mathscr{A}_{2, \Gamma_{\text {rem }}}=0 .
$$

Hence $\delta \mathscr{R}_{2, \Gamma}$ can be fully computed from tadpole integrals with one auxiliary scale $M^{2}$,

$$
\begin{aligned}
\delta \mathscr{R}_{2, \Gamma}=\tilde{\mathbf{K}}_{\mathrm{loc}} \mathscr{A}_{2, \Gamma}= & \tilde{\mathbf{K}}_{\mathrm{loc}} \prod_{n=1}^{3} \mathbf{S}_{X_{n}}^{(n)} \mathscr{A}_{2, \Gamma}=\left(\prod_{n=1}^{3} \mathbf{S}_{X_{n}}^{(n)} \overline{\mathscr{A}}_{2, \Gamma}+\sum_{i} \delta Z_{1, \gamma_{i}} \cdot \mathbf{S}_{X_{i}}^{(i)} \overline{\mathscr{A}}_{1, \Gamma / \gamma_{i}}\right) \\
& -\left(\prod_{n=1}^{3} \mathbf{S}_{X_{n}}^{(n)} \mathscr{A}_{2, \Gamma}+\sum_{i}\left(\delta Z_{1, \gamma_{i}}+\delta \tilde{Z}_{1, \gamma_{i}}+\delta \mathscr{R}_{1, \gamma_{i}}\right) \cdot \mathbf{S}_{X_{i}}^{(i)} \mathscr{A}_{1, \Gamma / \gamma}\right) .
\end{aligned}
$$

Here we use that the operators $\mathbf{S}_{X_{i}}^{(i)}$ fully capture the UV counterterms and rational terms in the sub-diagrams due to the definition (3.12) of the order of the tadpole decomposition, i.e.

$$
\mathbf{K}\left(\mathbf{S}_{X_{j}}^{(j)} \mathbf{S}_{X_{k}}^{(k)} \overline{\mathscr{A}}_{1, \gamma_{i}}\right)=-\delta Z_{1, \gamma_{i}}, \quad \tilde{\mathbf{K}}\left(\mathbf{S}_{X_{j}}^{(j)} \mathbf{S}_{X_{k}}^{(k)} \mathscr{A}_{1, \gamma_{i}}\right)=\delta \mathscr{R}_{1, \gamma_{i}} .
$$

Because of the exactness of the tadpole decomposition (3.13) and (3.17), the result is independent of $M^{2}$ and polynomial in external momenta and internal masses, and hence indeed a rational term. A sample diagram and its renormalisation in $D$ dimensions as well as its computation via the master formula (1.4) are depicted in Fig. 3.

\section{Results for QED}

In this section we present the two-loop rational terms in QED. They are calculated with QGRAF [13], Q2E and EXP [14,15] and MATAD [16]. We start from the Lagrangian

$$
\mathscr{L}_{Q E D}=\bar{\psi}\left(i \gamma^{\mu} D_{\mu}-m\right) \psi-\frac{1}{4} F_{\mu v} F^{\mu \nu}-\frac{1}{2 \lambda}\left(\partial^{\mu} A_{\mu}\right)^{2}, \quad D_{\mu}=\partial_{\mu}-i e A_{\mu}
$$

\footnotetext{
${ }^{9}$ Again $i \mid j k$ is a partition of 123 .

${ }^{10}$ Note that the operators $\mathbf{S}$ and $\mathbf{F}$ either fully reconstruct UV counterterms and rational terms or give zero, e.g. $\mathbf{K}\left(\mathbf{S}_{X_{j}}^{(j)} \mathbf{S}_{X_{k}}^{(k)} \overline{\mathscr{A}}_{1, \gamma_{i}}\right)=-\delta Z_{1, \gamma_{i}}$ and $\mathbf{K}\left(\mathbf{S}_{X_{j}}^{(j)} \mathbf{F}_{X_{k}}^{(k)} \overline{\mathscr{A}}_{1, \gamma_{i}}\right)=0$ and $\mathbf{K}\left(\mathbf{F}_{X_{j}}^{(j)} \mathbf{F}_{X_{k}}^{(k)} \overline{\mathscr{A}}_{1, \gamma_{i}}\right)=0$.
} 


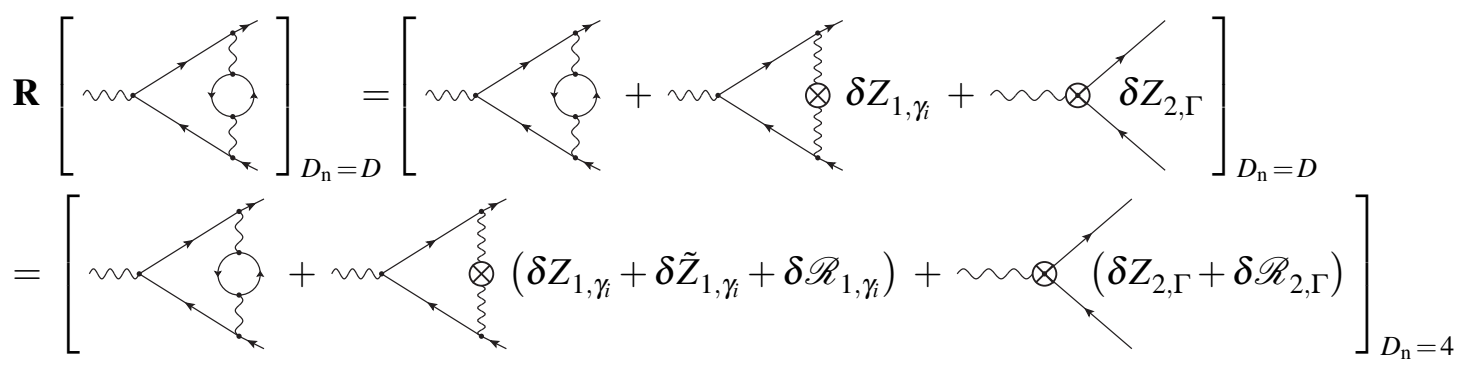

Figure 3: Graphical representation of the renormalisation formula (first line) in $D$ dimensions for a twoloop QED diagram with a single sub-divergence, and the master formula (second line) to compute it from four-dimensional amplitudes with universal counterterm insertions.

and set the fermion mass $m$ and the gauge parameter $\lambda=1$. The rational terms and the additional counterterm $\tilde{Z}$ for the photon self-energy are

$$
\begin{array}{ll}
\delta \mathscr{R}_{1, e}=\frac{i e^{2}}{16 \pi^{2}}[-1] \not p, & \delta \mathscr{R}_{2, e}=\frac{i e^{4}}{\left(16 \pi^{2}\right)^{2}}\left[\frac{19}{18 \varepsilon}+\frac{247}{108}\right] \not p \\
\delta \mathscr{R}_{1, \gamma}^{\mu v}=\frac{i e^{2}}{16 \pi^{2}}\left[\frac{2}{3} p^{2}\right] g^{\mu v}, & \delta \mathscr{R}_{2, \gamma}^{\mu v}=\frac{i e^{4}}{\left(16 \pi^{2}\right)^{2}}\left[P^{\mu v}\left(\frac{2}{3 \varepsilon}-\frac{71}{18}\right)+g^{\mu v} p^{2}\left(-\frac{11}{12}\right)\right] \\
\delta \tilde{Z}_{1, \gamma}^{\mu v}=\frac{i e^{2}}{16 \pi^{2}}\left[\frac{2}{3 \varepsilon} \tilde{p}^{2}\right] g^{\mu \nu} & \delta \mathscr{R}_{1, e e \gamma}=\frac{i e^{3}}{16 \pi^{2}}[-2] \gamma^{\mu},
\end{array}
$$

with $P^{\mu v}=p^{\mu} p^{v}-g^{\mu v} p^{2}$ and $G^{\mu v \rho \sigma}=g^{\mu v} g^{\rho \sigma}+g^{\mu \rho} g^{v \sigma}+g^{\mu \sigma} g^{v \rho}$. We find that the two-loop $\tilde{N}$-contributions are indeed polynomial in the external momentum $p$ and hence are rational terms. The full $m$ and $\lambda$-dependence of these terms is presented in [1].

\section{Conclusions}

We have presented an extension of the renormalisation procedure for $D$-dimensional amplitudes at two-loop level, such that the numerators of all Feynman diagrams can be constructed in four dimensions. We showed that the contributions stemming from the interplay of $(D-4)$ dimensional numerator with UV divergences can be reconstructed through a finite set of local counterterms, and presented this set for the case of QED. This constitutes an important building block for numerical two-loop calculations.

\section{Acknowledgments}

We thank J.-N. Lang for useful discussions. S.P., H.Z., M.Z. acknowledge support from the Swiss National Science Foundation (SNF) under contract BSCGI0-157722. M.Z. acknowledges 
support by the Swiss National Science Foundation (Ambizione grant PZ00P2-179877).

\section{References}

[1] S. Pozzorini, H. Zhang, and M. F. Zoller, Rational Terms at Two Loops, in preparation.

[2] W. E. Caswell and A. D. Kennedy, Simple approach to renormalization theory, Phys. Rev. D 25 (1982), no. 2 392-408.

[3] F. Buccioni, J.-N. Lang, J. M. Lindert, P. Maierhöfer, S. Pozzorini, H. Zhang, and M. F. Zoller, OpenLoops 2, Eur. Phys. J. C79 (2019), no. 10 866, [arXiv: 1907.1307].

[4] A. Denner, J.-N. Lang, and S. Uccirati, Recola2: REcursive Computation of One-Loop Amplitudes 2, Comput. Phys. Commun. 224 (2018) 346-361, [arXiv: 1711. 0738].

[5] G. Bevilacqua, M. Czakon, M. V. Garzelli, A. van Hameren, A. Kardos, C. G. Papadopoulos, R. Pittau, and M. Worek, HELAC-NLO, Comput. Phys. Commun. 184 (2013) 986-997, [arXiv:1110.1499].

[6] V. Hirschi, R. Frederix, S. Frixione, M. V. Garzelli, F. Maltoni, and R. Pittau, Automation of one-loop QCD corrections, JHEP 05 (2011) 044, [arXiv: 1103.0621$].$

[7] G. Ossola, C. G. Papadopoulos, and R. Pittau, On the Rational Terms of the one-loop amplitudes, JHEP 05 (2008) 004, [arXiv: 0802 . 1876].

[8] P. Draggiotis, M. V. Garzelli, C. G. Papadopoulos, and R. Pittau, Feynman Rules for the Rational Part of the QCD 1-loop amplitudes, JHEP 04 (2009) 072, [arXiv: 0903.0356 ].

[9] M. V. Garzelli, I. Malamos, and R. Pittau, Feynman rules for the rational part of the Electroweak 1-loop amplitudes, JHEP 01 (2010) 040, [arXiv: 0910 . 3130]. [Erratum: JHEP10,097(2010)].

[10] R. Pittau, Primary Feynman rules to calculate the epsilon-dimensional integrand of any 1-loop amplitude, JHEP 02 (2012) 029, [arXiv: 1111.4965].

[11] M. Misiak and M. Munz, Two loop mixing of dimension five flavor changing operators, Phys. Lett. B344 (1995) 308-318, [hep-ph/ 9409454$].$

[12] K. G. Chetyrkin, M. Misiak, and M. Münz, Beta functions and anomalous dimensions up to three loops, Nucl. Phys. B518 (1998) 473-494, [hep-ph/9711266].

[13] P. Nogueira, Automatic Feynman graph generation, J. Comput. Phys. 105 (1993) 279-289.

[14] T. Seidensticker, Automatic application of successive asymptotic expansions of Feynman diagrams, in 6th International Workshop on New Computing Techniques in Physics Research (AIHENP 99) Heraklion, Crete, Greece, April 12-16, 1999, 1999. hep-ph/9905298.

[15] R. Harlander, T. Seidensticker, and M. Steinhauser, Complete corrections of Order alpha alpha-s to the decay of the Z boson into bottom quarks, Phys.Lett. B426 (1998) 125-132, [hep-ph/9712228].

[16] M. Steinhauser, MATAD: A program package for the computation of massive tadpoles, Comput. Phys. Commun. 134 (2001) 335-364, [hep-ph/ 000902 9].

[17] A. Bredenstein, A. Denner, S. Dittmaier, and S.Pozzorini, $N L O Q C D$ corrections to t anti-t $b$ anti-b production at the LHC: 1. Quark-antiquark annihilation, JHEP 08 (2008) 108, [arXiv:0807.1248]. 